Trauma Berufskrankh 2007 - 9 [Suppl 2]:S132-S136 DOI 10.1007/s10039-006-1203-2

Online publiziert: 24. Januar 2007

๑) Springer Medizin Verlag 2007
S. Kübler · J. Jähne

Klinik für Allgemein- und Viszeralchirurgie, Schwerpunkt für onkologische und endokrine Chirurgie, Diakoniekrankenhaus Henriettenstiftung gGmbH, Hannover

\title{
Abdominelle Verletzungen des Retroperitoneums und der Gefäße
}

\author{
Abdominelles Kompartmentsyndrom
}

\section{Epidemiologie}

Unfälle mit Beteiligung von Abdomen, Becken, Nieren oder großen Gefäßen machen nach einer Statistik des Hauptverbandes der gewerblichen Berufsgenossenschaften (HVBG) aus dem Jahr 2004 mit einer Zahl von 10.626 nur einen kleinen Anteil von etwa 10\%, bezogen auf die Gesamtzahl der insgesamt 1.112.210 gemeldeten Arbeits- und Wegeunfälle, aus. Dabei handelt es sich zu einem Großteil um oberflächliche Prellungen ohne bedeutende Organverletzungen. Im Jahr 2004 waren nur in 424 Fällen abdominelle oder retroperitoneale Organe direkt betroffen. Bezogen auf die Gesamtzahl der gemeldeten Verletzungen, kommen diese mit einer Häufigkeit von ca. 0,4\%o, bezogen auf die Anzahl der gemeldeten Abdominaltraumata mit ca. 4\%, außerordentlich selten vor [3].

In ca. 10\% der Fälle werden die Organverletzungen durch penetrierende Verletzungen hervorgerufen, im Vordergrund steht jedoch die Einwirkung stumpfer Gewalt mit einem Anteil von ca. 90\% (- Tab. 1; $[1,2,3])$. Die Verletzungen werden hierbei durch plötzlich auftretende Dezelerationskräfte bei Aufprall eines intraabdominellen oder retroperitonealen Organs gegen die Wirbelsäule oder das Becken oder eine auf gleiche Weise entstehende plötzliche Erhöhung des intraabdominellen Drucks hervorgerufen und werden in ca. zwei Drittel der Fälle durch Verkehrsunfälle verursacht. Folge sind Organkontusionen oder -zerreißungen oder das Platzen eines Hohlorgans. Aufgrund der hierbei meist großen Krafteinwirkung sind Kombinationsverletzungen unter Einbeziehung auch knöcherner Strukturen häufig. Aufgrund des Überwiegens von Kombinationsverletzungen mit mehreren betroffenen Organen und insbesondere knöchernen Begleitverletzungen mit daraus resultierenden erheblichen Auswirkungen auf den Gesamtorganismus müssen diagnostische und entsprechende therapeutische Maßnahmen zuallererst am Gesamtzustand des Patienten ausgerichtet werden $[1,2]$.

\section{Betroffene Organe}

In erster Linie betroffene Organe sind die Milz und die Leber, unter den retroperitonealen Organen sind Verletzungen der Niere mit weitem Abstand vor Verletzung des Duodenums und des Pankreas führend (• Tab. $1 ;[2,3,4])$.

\section{Nierenverletzungen}

Die Einteilung der Verletzungen auf die einzelnen Organe bezogen erfolgt zumeist nach von Moore et al 1989 publizierten, von der American Association for the Surgery of Trauma (AAST) übernommenen Klassifikationen [4, 5]. Die hierfür grundlegende Diagnostik besteht zumeist in der Durchführung einer abdominellen Computertomographie, bei Verdacht auf Nierenverletzungen auch mit Spätaufnahmen zur Uretherendarstellung $[1,2,6]$. Hierbei können oberflächliche Verletzungen mit Ausbildung subkapsulärer Hämatome bei intakter Nierenkapsel sowie oberflächliche Parenchymeinrisse und tiefer gehende Parenchymeinrisse ohne Extravasation von tiefer gehenden Verletzungen mit Urinextravasation oder einer kompletten Nierenzerreißung mit Hiluszerreißung und Devaskularisation unterschieden werden. Die Therapie der solitären Nierenverletzungen

\section{Tab. 1 Epidemiologie und Verletzungsmechanismus}

\begin{tabular}{|c|c|c|c|}
\hline \multicolumn{4}{|l|}{ Epidemiologie $^{a}$} \\
\hline \multicolumn{3}{|c|}{ Abdominelle/retroperitoneale Organverletzungen } & 424 \\
\hline \multirow[t]{2}{*}{ Bezogen auf } & \multicolumn{2}{|c|}{ Meldepflichtige Unfälle gesamt $(n=1.112 .210)$} & $0,38 \% 0$ \\
\hline & \multicolumn{2}{|c|}{ Abdominaltraumata $(n=10.626)$} & $3,8 \%$ \\
\hline \multirow{2}{*}{\multicolumn{2}{|c|}{ Verletzungsmechanismus $^{b}$}} & Stumpf & Penetrierend \\
\hline & & $90 \%$ & $10 \%$ \\
\hline \multirow[t]{3}{*}{ Retroperitoneum } & Niere & $24 \%$ & $3-5 \%$ \\
\hline & Duodenum & $1-3 \%$ & $3-5 \%$ \\
\hline & Pankreas & $1-3 \%$ & $3-5 \%$ \\
\hline
\end{tabular}


sollte stadienadaptiert und insbesondere bei geringergradigen Verletzungen oder solitärer Nierenverletzung nach Möglichkeit unter Hinzuziehung eines Fachurologen erfolgen $[1,2,7]$. Hierbei stellt der Organerhalt das vorrangige Behandlungsziel dar (• Tab. 2)

Bei Verletzungen der Grade 1-3 kann hierbei konservativ vorgegangen werden, ebenfalls überwiegend konservativ erfolgt die Behandlung des Stadiums IV, wobei hier ggf. eine Harnleiterschienung notwendig sein kann. Im Stadium V mit ausgedehnter Parenchymzerreißung oder Hiluszerreißung ist eine operative Versorgung unumgänglich. Die therapeutischen Möglichkeiten reichen hier von einer partiellen Resektion zum Parenchymerhalt bis zu einer Nephrektomie. Die Notwendigkeit zur Nephrektomie kann insbesondere auch durch den Gesamtzustand des Patienten mit schwerwiegenden, ausgeprägteren Verletzungen gegeben sein, vorrangiges Behandlungsziel ist die Stabilisierung des Patienten $[1,2,7]$.

\section{Duodenumverletzungen}

Die Verletzungen des Duodenums werden ebenfalls seit 1990 nach Verletzungsmuster und Ausprägung der Verletzungen in Schweregrade nach Moore et al. [5] eingeteilt (• Tab. 3).

Die Behandlungsoptionen reichen von einer einfachen Übernähung über die Direktnaht oder die Segmentresektion bis zum Verschluss eines Defektes durch eine nach Y-Roux gebildete Jejunalschlinge. Die Ultima Ratio bei massiven ZerreiBungen des duodenopankreatischen Komplexes stellt die partielle oder vollständige Pankreatikoduodenektomie dar $([1,2,5,7] ; \mathbf{0}$ Tab. 3).

\section{Pankreasverletzungen}

Auch bei den Pankreasverletzungen hat sich die Schweregradeinteilung nach Moore et al. [5] durchgesetzt, eine alternative Einteilung nach Lucas findet demgegenüber kaum Verwendung. Wesentliche Bedeutung für Prognose und Therapie der Pankreasverletzungen hat hier das Vorliegen von Gangverletzungen. Zentral in der Therapie stehen Débridement und insbesondere die adäquate Drainage

Trauma Berufskrankh 2007 · 9 [Suppl 2]:S132-S136 DOI 10.1007/s10039-006-1203-2

(c) Springer Medizin Verlag 2007

S. Kübler · J. Jähne

Abdominelle Verletzungen des Retroperitoneums und der Gefäße. Abdominelles Kompartmentsyndrom

\section{Zusammenfassung}

Verletzungen von im Retroperitoneum gelegenen Organen und den großen Gefäßen sind als Folge von Arbeitsunfällen selten, der Verletzungsmechanismus ist überwiegend stumpf. Die Schweregradeinteilung dieser Verletzungen erfolgt nach einer durch Moore et al. eingeführten, auf die einzelnen Organe bezogenen Klassifikation. Die Therapie der jeweiligen Verletzungen erfolgt schweregradabhängig und reicht von konservativen über interventionelle Maßnahmen bis hin zur Teilresektion oder Resektion der betroffenen Organe. Leichtere Verletzungen der Organe des Retroperitoneums gehen mit einer relativ guten Prognose einher. Demgegenüber zeigen schwerwiegendere Verletzungen der Organe des Retroperitoneums eine hohe Morbidität und Mortalität. Hierfür ist zum Teil die

\section{Abdominal injuries of the retroperitoneum and the blood vessels. Abdominal compartment syndrome}

\section{Abstract}

Injuries of organs of the retroperitoneum and the large blood vessels are rare as the consequence of work-related accidents or traffic accidents; the injury mechanism is predominantly blunt. The severity of the individual organ injuries is classified according to injury scores first described by Moore et al. Therapeutic measures cover a broad spectrum of modalities from conservative measures only, interventional therapies, and partial or complete resection of the organs involved. Lighter injuries of the retroperitoneal organs have a relatively good prognosis. In contrast to this, more serious injuries of the retroperitoneal organs show a high morbidity and mortality. Partly responsible for this is an unphysiologic increase of the intra-abdominal pressure, the abdominal compartment syndrome (ACS). This clinical entity ap- als Folge ausgedehnter Verletzungen des Abdomens und des Retroperitoneums auftretende, unphysiologische Erhöhung des intraabdominellen Druckes, das abdominelle Kompartmentsyndrom (ACS), verantwortlich. Dieses häufig verkannte Syndrom mit seinen Auswirkungen auf Herz-Kreislauf-, Lungen und Nierenfunktion ist unbehandelt mit einer hohen Morbidität und Mortalität vergesellschaftet. Die Therapie besteht in der Volumengabe und Faszieneröffnung zur abdominellen Dekompression.

\section{Schlüsselwörter}

Abdominelle Verletzungen · Abdominelles Kompartmentsyndrom - Retroperitoneum . Große Gefäße · Therapie pearing as the consequence of extensive injuries of the abdomen and the retroperitoneum is a frequently misjudged and misdiagnosed syndrome with serious impairment of heart, lung, and kidney function. Therapeutic measures range from volume substitution to abdominal decompression by opening of the abdominal compartment. The fascia is then kept open by various methods to achieve relief of intra-abdominal hypertension for a longer duration. Left untreated, this syndrome leads to a high morbidity and mortality, but even with adequate treatment, morbidity and mortality remain significant.

\section{Keywords}

Abdominal injuries · Abdominal compartment syndrome $\cdot$ Retroperitoneum $\cdot$ Large blood vessels . Therapy 


\section{Bauchverletzungen}

\begin{tabular}{|c|c|c|}
\hline Grad & Art & Therapie \\
\hline I & $\begin{array}{l}\text { Nierenkontusion, subkapsuläres Hämatom bei intakter } \\
\text { Kapsel }\end{array}$ & Konservativ \\
\hline II & $\begin{array}{l}\text { Parenchymeinriss }<1 \mathrm{~cm} \text { tief ohne Beteiligung des Nieren- } \\
\text { beckenkelchsystems oder tiefer Markschichten }\end{array}$ & \\
\hline III & Parenchymeinriss $>1 \mathrm{~cm}$ tief ohne Extravasation & \\
\hline IV & $\begin{array}{l}\text { Parenchymeinriss bis ins Nierenkelchsystem ohne segmen- } \\
\text { tale GefäßverletzungExtravasation, segmentaler Funkti- } \\
\text { onsausfall }\end{array}$ & $\begin{array}{l}\text { Konservativ, } \\
\text { ggf. Harnleiter- } \\
\text { schienung }\end{array}$ \\
\hline V & $\begin{array}{l}\text { Nierenzerreißung ohne GefäßstielverletzungKomplett } \\
\text { fragmentierte Niere, HiluszerreißungDevaskularisation }\end{array}$ & $\begin{array}{l}\text { Operative Versorgung } \\
\text { ggf. Resektion }\end{array}$ \\
\hline
\end{tabular}

\begin{tabular}{|c|c|c|}
\hline Grad & Art der Verletzung & Therapie \\
\hline I & $\begin{array}{l}\text { Hämatom oder Einriss eines einzelnen Duodenalseg- } \\
\text { mentes ohne Perforation }\end{array}$ & $\begin{array}{l}\text { Konservativ, } \\
\text { Übernähung }\end{array}$ \\
\hline II & $\begin{array}{l}\text { Hämatom oder Ruptur in einem Duodenalsegment }<50 \% \\
\text { der Zirkumferenz }\end{array}$ & Direkte Naht \\
\hline III & $\begin{array}{l}\text { Ruptur in } 50-75 \% \text { der Zirkumferenz in Pars II duodeni oder } \\
\text { in }>50 \% \text { der Zirkumferenz in einem Duodenalsegment }\end{array}$ & \multirow[t]{2}{*}{$\begin{array}{l}\text { Segmentresektion } \\
\text { Y-Roux-Jejunalschlinge }\end{array}$} \\
\hline IV & $\begin{array}{l}\text { Ruptur in }>75 \% \text { der Zirkumferenz in Pars II duodeni oder } \\
\text { mit Beteiligung der Papilla Vateri oder distalem Gallengang }\end{array}$ & \\
\hline V & $\begin{array}{l}\text { Massive Zerreißung des duodenopankreatischen } \\
\text { Komplexes oder Devaskularisierung des Duodenums }\end{array}$ & $\begin{array}{l}\text { Pankreatikoduoden- } \\
\text { ektomie }\end{array}$ \\
\hline
\end{tabular}

Tab. 4 Pankreasverletzung (AAST, [5]

\begin{tabular}{lll} 
Grad & $\begin{array}{l}\text { Art der Verletzung } \\
\text { Geringgradige Kontusion oder oberflächliche Parenchym- } \\
\text { verletzung ohne Gangbeteiligung }\end{array}$ & $\begin{array}{l}\text { Therapie } \\
\text { Débridement, } \\
\text { Übernähung, Drainage }\end{array}$ \\
II & $\begin{array}{l}\text { Ausgedehnte Kontusion oder Parenchymverletzung ohne } \\
\text { Gangbeteiligung }\end{array}$ & \\
III & $\begin{array}{l}\text { Distale Ruptur oder Parenchymverletzung mit Gangbetei- } \\
\text { ligung }\end{array}$ & $\begin{array}{l}\text { Distale Resektion, Naht, } \\
\text { Débridement, Drainage }\end{array}$ \\
IV & $\begin{array}{l}\text { Proximale Ruptur oder Parenchymverletzung mit Beteili- } \\
\text { gung der Papille }\end{array}$ & $\begin{array}{l}\text { Distale Resektion, pro- } \\
\text { ximale Übernähung, } \\
\text { distale Drainage mit } \\
\text { V }\end{array}$ \\
Massive Zertrümmerung des Pankreaskopfes & $\begin{array}{l}\text { Y-Roux-Schlinge, Duo- } \\
\text { denopankreatektomie }\end{array}$ \\
\hline
\end{tabular}

Tab. 5 Abdominelles Kompartmentsyndrom (WSAC, [12])

\begin{tabular}{|c|c|c|}
\hline \multirow[t]{3}{*}{ Kreislaufinsuffizienz } & \multicolumn{2}{|l|}{$\mathrm{HZV} \downarrow$} \\
\hline & \multicolumn{2}{|l|}{ ZVD个 } \\
\hline & \multicolumn{2}{|l|}{ Venöser Rückfluss $\downarrow$} \\
\hline \multirow[t]{3}{*}{ HyperkapnieHypoxie } & \multicolumn{2}{|l|}{ Beatmungsdruck $\uparrow$} \\
\hline & \multicolumn{2}{|l|}{ Compliance $\downarrow$} \\
\hline & \multicolumn{2}{|l|}{ FRC $\downarrow$} \\
\hline \multirow[t]{2}{*}{ Oligurie/Anurie } & \multicolumn{2}{|l|}{ GFR $\downarrow$} \\
\hline & \multicolumn{2}{|l|}{ Nierenperfusion $\downarrow$} \\
\hline \multicolumn{3}{|c|}{ Bestimmung des intraabdominellen Druckes durch Blasendruckmessung } \\
\hline Grad & IAD [mmHg] & Therapie \\
\hline I & $10-15$ & Bilanzierung \\
\hline II & $16-25$ & Volumentherapie \\
\hline III & $26-35$ & Dekompression \\
\hline IV & $>35$ & Reexploration \\
\hline
\end{tabular}

sowie die entsprechende Ableitung oder Versorgung von Gangverletzungen durch Kombinationen von Resektion und innerer Drainage durch nach Y-Roux gebildete Jejunalschlingen in Abhängigkeit von Lokalisation und Art der Gangverletzung ([1, 2, 7]; - Tab. 4).

Komplexere Verletzungen entsprechend dem Grad V der Einteilung sowohl des Pankreaskopfes als auch des Duodenums treten aufgrund der hierzu notwendigen massiven Krafteinwirkung in $20-$ $50 \%$ der Fälle gemeinsam auf und sind dann in $50-98 \%$ der Fälle mit weiteren Organverletzungen vergesellschaftet. Die Komplikationsrate dieser Verletzungen mit bis zu $60 \%$ und die Mortalitätsrate mit ca. $20 \%$ sind außerordentlich hoch. Insbesondere die in Fällen ausgedehnter Verletzungen des Pankreaskopfes oder Zerreißung des duodenopankreatischen Komplexes notwendige Duodenopankreatektomie ist bei Durchführung im Akutfall mit einer außerordentlich hohen Mortalität von ca. $30 \%$ vergesellschaftet $[2,5,7,8$, 9]. Die Versorgung solcher Verletzungen sollte in Zentren, in denen operative Eingriffe der Bauchspeicheldrüse regelmäßig durchgeführt werden, erfolgen. Ergibt sich eine entsprechende Diagnose im Rahmen einer Exploration bei instabilem Patienten, so sollte aufgrund der Komplexität dieser Verletzungen und der zur Versorgung notwendigen Therapiemaßnahmen nach Stabilisierung und Drainageeinlage eine Verlegung in ein solches Zentrum erfolgen. Gleiches gilt bei Diagnosestellung durch Computertomographie und ggf. Endoskopie [7, 8, 9].

\section{Hämatome}

Als Folge stumpfer Gewalteinwirkung kann es zur Ausbildung ausgedehnter retroperitonealer Hämatome kommen. Diese können nach ihrer Lokalisation verschiedenen Zonen zugeordnet werden (• Abb. 1).

Hämatome der zentralen Zone I in Abdomenmitte werden zumeist durch Verletzungen der hier verlaufenden großen Gefäße oder aber durch Verletzungen von Pankreas und Duodenum sowie der Lebervenen hervorgerufen, sie gehen zumeist mit einer hämodynamischen Instabilität einher, eine operative Explora- 
tion ist hier obligat. Dabei muss zur adäquaten Exposition zumeist eine mediale, viszerale Rotation durchgeführt werden. Hierzu wird das Intestinalpaket nach lateraler Mobilisation nach links abgewälzt. Die Überlebensrate bei Gefäßverletzungen liegt je nach Art der Verletzung und den betroffenen Gefäßen zwischen 8 und $40 \%$. Rekonstruktive Maßnahmen reichen hier von Übernähungen bis zum Gefäßersatz und sind situationsabhängig $([1,2,10] ; \bullet$ Abb. 1)

Hämatome der Flankenzone (Zone II) werden meist durch Nierenverletzung verursacht. Die Therapie richtet sich nach dem Ausmaß der Nierenverletzungen, wobei hier zumeist die Diagnose durch Computertomographie erfolgt. Eine eindeutige Indikation zur operativen Freilegung besteht bei expandierendem, rupturierendem oder pulsierendem Hämatom in diesem Bereich.

Hämatome in Zone III werden meist durch Beckenfrakturen oder eine mehr oder weniger ausgedehnte Zerreißung der knöchernen Strukturen des Beckens hervorgerufen. Vordringlich in der Therapie ist hier zunächst die Stabilisierung der Beckenfraktur zumeist durch äußere Schienung und Kompression. Bei Blutungspersistenz trotz Stabilisierung der Beckenfraktur sollte zunächst in Abhängigkeit vom Gesamtzustand des Patienten eine Angiographie und ggf. selektive Embolisation erfolgen. Eine eindeutige Indikation zur operativen Freilegung besteht bei expandierenden, rupturierenden oder pulsierenden Hämatomen oder bei auch unter maximalen konservativen Maßnahmen weiterhin instabilem Patienten $[1,2,10]$.

\section{Größere Blutverluste}

Insgesamt können ausgedehnte, schwerwiegende Verletzungen, hier insbesondere Gefäßverletzungen, größere Blutverluste hervorrufen. Diese wiederum führen zu einem Circulus vitiosus aus Koagulopathie, Hypothermie und Azidose mit zunehmender Instabilität des Patienten. Hier ist aufgrund des kompromittierten Allgemeinzustandes in der Regel ein therapeutisches Vorgehen entsprechend den zentralen Behandlungsgrundlagen der „damage control surgery" notwendig. Diese beinhalten einerseits die Blutungskontrolle, an-

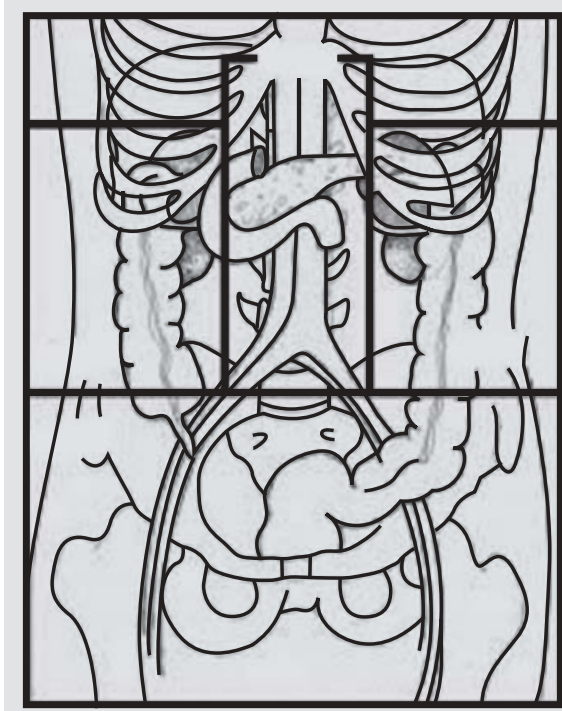

Mediale viszerale Rotation

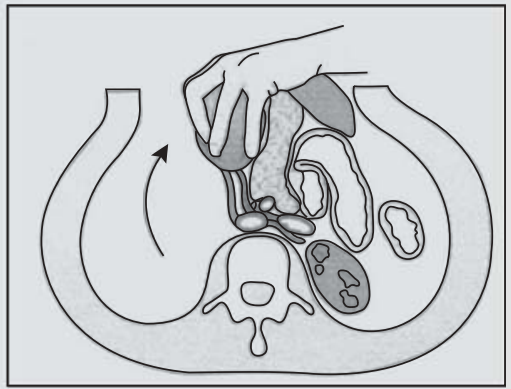

Abb. $1 \Delta$ Retroperitoneale Hämatome
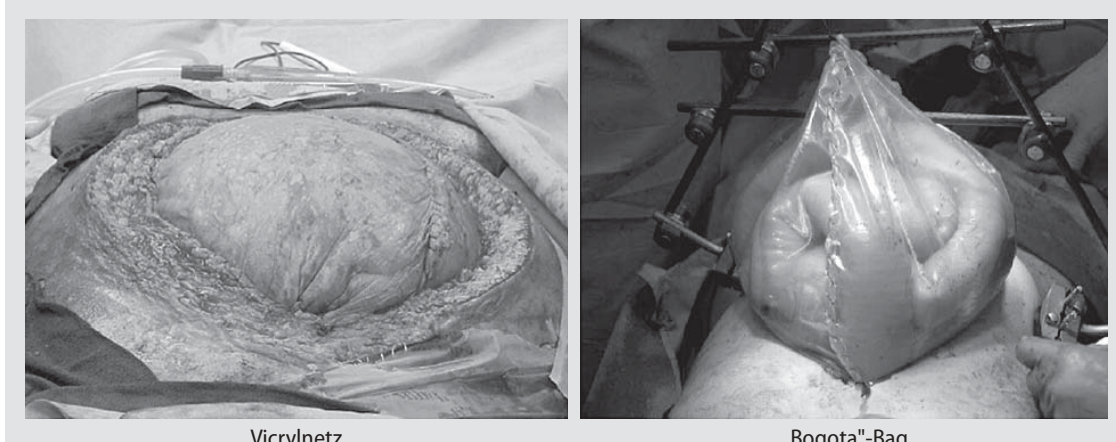

Vicrylnetz

"Bogota"-Bag

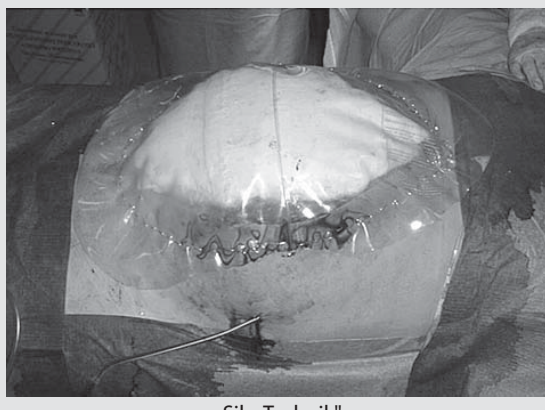

"Silo-Technik"

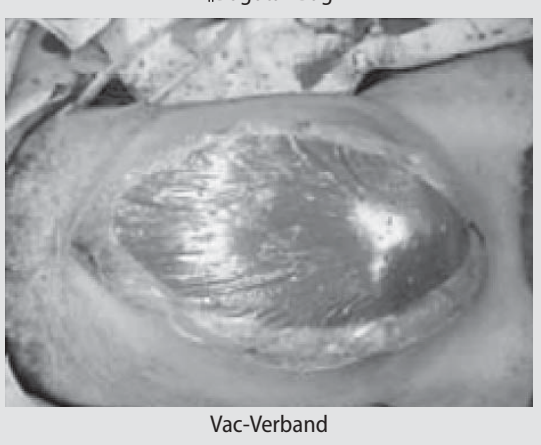

Abb. $2 \Delta$ Dekompression

dererseits die Vermeidung von Kontamination bei zusätzlichen Darmverletzungen durch raschen Verschluss eröffneter Darmlumina. Ziel ist es, möglichst schnell die minimal notwendigen operativen Schritte zur Blutungskontrolle und Kontaminationsvermeidung durchzuführen um den Patienten dann zunächst durch intensivmedizinische Maßnahmen auf der Intensivstation zu stabilisieren. Komplexere operative Schritte und rekonstruktive Maßnahmen, z. B. zur Wiederherstellung der Darmkon- tinuität, werden anschließend nach Überwindung von Koagulopathie, Azidose und Hypothermie durchgeführt.

\section{Abdominelles Kompartmentsyndrom}

Bei schwerstverletzten Patienten mit ausgedehnten intraperitonealen oder retroperitonealen Organverletzungen, die die Initialphase überleben, kommt es häufig zur Entwicklung eines abdominellen 
Kompartmentsyndroms $[11,12,13,14,15$, 16].

Das abdominelle Kompartmentsyndrom ist gekennzeichnet durch eine Kreislaufinsuffizienz bei erhöhtem zentralvenösem Druck, Hyperkapnie und Hypoxie bei erhöhtem Beatmungsdruck sowie Oligurie oder Anurie (• Tab. 5). Diese Symptome sind Ausdruck eines erhöhten intraabdominellen Druckes, der durch die Verminderung des venösen Rückflusses sowie die verminderte Atemexkursion des Zwerchfelles zu den aufgeführten klinischen Symptomen führt. Das ACS ist nach entsprechender Therapie reversibel. Der intraabdominelle Druck lässt sich durch standardisierte Bestimmung des Blaseninnendruckes bei mit $50 \mathrm{ml}$ Kochsalzlösung aufgefüllter Blase und Anschluss des Blasenkatheters an einen Transducer oder aber an ein System, wie es zur Bestimmung des zentralvenösen Druckes benutzt wird, abschätzen.

Die Einteilung in Stadien und entsprechende Therapie kann in Abhängigkeit von den hierbei gemessenen Druckwerten erfolgen. Bei Bestimmung intraabdomineller Druckwerte sollte ab einem Druckwert von mehr als $25 \mathrm{mmHg}$ eine operative Dekompression durch Faszieneröffnung erfolgen. Generell gilt jedoch, dass bei Vorliegen der angeführten klinischen Symptome ohne Besserungszeichen trotz Volumentherapie bei Unklarheit eine operative Druckentlastung erfolgen sollte (• Tab. 5).

Die Faszien müssen hier zur dauerhaften Dekompression offen belassen werden, hierzu stehen verschiedene Verfahren zur Verfügung.

Dies sind zum einen die Einnaht eines Vicrylnetzes, wobei dieses locker an die Faszienränder oder aber an den Hautrand fixiert werden kann. Alternative Verwendung findet eine sog. „Bogota-Bag“ als an den Faszienrändern fixierte Plastikfolie, z. B. aus einem sterilen Infusionsbeutel. Weiterhin Verwendung finden VaccumVerbände oder die Silo-Technik durch Abdecken des Intestinums mittels feuchter Bauchtücher und lockere Fixation der Bauchtücher durch aufgeklebte Op-siteFolien (• Abb. 2).

Der Faszienverschluss kann nach entsprechender Erholung entweder kurzfristig oder aber zeitverzögert nach Jahren mit ähnlichen Erfolgsraten für die Ausbildung von Narbenhernien und Wundinfekten erfolgen. Prinzipiell ist für einen Entscheid zum Faszienverschluss der Gesamtzustand des Patienten ausschlaggebend $[11,12,13,14,15,16]$.

\section{Fazit für die Praxis}

Verletzungen retroperitonealer Organe sind mit einer Häufigkeit von $3 \%$ seltene Verletzungen. Ausgeprägtere Schäden betreffen häufig mehrere Organsysteme und gehen mit Kreislaufinstabilität einher, aufgrund ihrer Komplexität sind sie häufig schwer zu versorgen, die Morbidität mit ca. $60 \%$ und die Mortalität zwischen 20 und $90 \%$ sind entsprechend hoch. Die Initialversorgung der instabilen Patienten folgt den Prinzipien der "damage control surgery" mit den vorrangigen Behandlungszielen Blutungskontrolle und Kontaminationsvermeidung. Zur definitiven Versorgung sollte die Verlegung in entsprechende Zentren erwogen werden. Bei komplexen, ausgedehnteren Verletzungen des Retroperitoneums besteht überdies ein hohes Risiko für das Auftreten eines abdominellen Kompartmentsyndroms mit seinen entsprechenden klinischen Zeichen. Wesentlich ist bei Verdacht auf das Vorliegen eines Kompartmentsyndroms die frühzeitige, weiterführende Diagnostik und insbesondere auch bei Unklarheiten die unverzügliche adäquate Therapie des Kompartmentsyndroms zumeist durch Dekompression.

\section{Korrespondierender Autor Dr. S. Kübler}

Klinik für Allgemein- und Viszeralchirurgie, Schwerpunkt für onkologische und endokrine Chirurgie, Diakoniekrankenhaus Henriettenstiftung gGmbH Marienstraße 72-90, 30171 Hannover stefan.kuebler@henriettenstiftung.de

Interessenkonflikt. Es besteht kein Interessenkonflikt. Der korrespondierende Autor versichert, dass keine Verbindungen mit einer Firma, deren Produkt in dem Artikel genannt ist, oder einer Firma, die ein Konkurrenzprodukt vertreibt, bestehen. Die Präsentation des Themas ist unabhängig und die Darstellung der Inhalte produktneutral.

\section{Literatur}

1. ACS Committee on Trauma (2004) Abdominal trauma. In: ATLS Student Course Manual, 7th edn. pp 131-150

2. Salomone JA, Salomone JP, Keim SM et al. (2006) Abdominal trauma, blunt. http://www.emedicine. com/emerg/topic1.htm

3. Hauptverband der gewerblichen Berufsgenossenschaften (HVBG) (2004) Referat „Statistik - Arbeits unfälle, Prävention"

4. Moore EE, Shackford SR, Pachter HL et al. (1989) Organ injury scaling: spleen, liver, and kidney. J Trauma 29(12): 1664-1666

5. Moore EE, Cogbill TH, Malangoni MA et al. (1990) Organ injury scaling, II: pancreas, duodenum, small bowel, colon, and rectum. J Trauma 30(11): 1427-1429

6. Goodacre B, van Sonnenberg E (2000) Radiologic evaluation of renal trauma. Intensive Care Med 15 90-98

7. Schumpelick V, Ambacher T, Riesener KP (1999) Aktuelle Therapie der Verletzungen von Colon und Retroperitoneum. Chirurg 70: 1269-1277

8. Bjerke HS, Dunn E, Talavera F et al. (2006) Pancreatic trauma. http://www.emedicine.com/emerg/ TRAUMA_AND_ORTHOPEDICS.htm

9. Klar E, Angelescu M, Richter G, Herfarth C (1999) Aktuelle Therapie bei Verletzungen des hepatobiliopankreatischen Kompartments. Chirurg 70: 1255-1268

10. Feliciano DV (2004) Injuries to the great vessels of the abdomen, ACS Surgery and principles. Wiley W. Suba, pp 1250-1261

11. Schein M, Wittmann DH, Aprahamian CC, Condon RE (1995) The abdominal compartment syndrome: the physiological and clinical consequences of elevated intra-abdominal pressure. J Am Coll Surg 180: 745-753

12. Burch JM, Moore EE, Moore FA et al. (1996) The abdominal compartment syndrome. Surg Clin North Am 76: 833-842

13. Bailey J, Shapiro MJ (2000) Abdominal compartment syndrome. Crit Care 4: 23-29

14. Fox VJ (1999) Temporary abdominal closure using an IV bag silo for severe trauma. AORN J 69: 560567

15. Ivatury RR, Diebel L, Porter JM et al. (1997) Intraabdominal hypertension and the abdominal compartment syndrome. Surg Clin North Am 77: 783 800

16. Howdieshell TR, Proctor CD, Sternberg E et al. (2004) Temporary abdominal closure followed by definitive abdominal wall reconstruction of the open abdomen. Am J Surg 188: 301-306 